\title{
BMJ Open Treatment with the anti-IgE monoclonal antibody omalizumab in women with asthma undergoing fertility treatment: a proof-of-concept study - The PRO-ART study protocol
}

Casper Tidemandsen (D) , ${ }^{1}$ Elisabeth Juul Gade, ${ }^{2}$ Charlotte Suppli Ulrik, ${ }^{1}$ Henriette Svarre Nielsen, ${ }^{3}$ Birgitte Sophie Oxlund-Mariegaard, ${ }^{4}$ Karsten Kristiansen, ${ }^{5}$ Nina La Cour Freiesleben, ${ }^{3}$ Bugge Nøhr, ${ }^{6}$ Hanne Udengaard, ${ }^{6}$ Vibeke Backer ${ }^{7}$

To cite: Tidemandsen C Juul Gade E, Ulrik CS, et al. Treatment with the antiIgE monoclonal antibody omalizumab in women with asthma undergoing fertility treatment: a proof-of-concept study-The PRO-ART study protocol. BMJ Open 2020;10:e037041. doi:10.1136/ bmjopen-2020-037041

- Prepublication history and supplemental material for this paper are available online. To view these files, please visit the journal online (http://dx.doi. org/10.1136/bmjopen-2020037041).

Received 16 January 2020 Revised 05 September 2020 Accepted 22 September 2020

D) Check for updates

(c) Author(s) (or their employer(s)) 2020. Re-use permitted under CC BY-NC. No commercial re-use. See rights and permissions. Published by BMJ.

For numbered affiliations see end of article.

Correspondence to Dr Casper Tidemandsen; casper.tidemandsen.02@ regionh.dk

\section{ABSTRACT}

Introduction Asthma is associated with prolonged time to pregnancy and a higher need for fertility treatment. However, the mechanism underlying this association remains incompletely understood. Previous research points to asthma-driven systemic inflammation also affecting the reproductive organs and thereby fertility. The aim of this study was to determine if treatment with omalizumab prior to fertility treatment will increase pregnancy rate among women with asthma by decreasing the systemic asthma-related inflammation and, by that, to provide insight into the underlying mechanisms.

Methods and analysis This is an ongoing prospective multicentre randomised controlled trial planned to enrol 180 women with asthma recruited from fertility clinics in Denmark. The patients are randomised 1:1 to either omalizumab or placebo. The primary endpoint is the difference in pregnancy rate confirmed with ultrasound at gestational week 7 of pregnancy. The secondary endpoints are change in sputum and blood eosinophil cell count, change in biomarkers, change in microbiota, together with rate of pregnancy loss, frequency of malformations, pre-eclampsia, preterm birth, birth weight, small for gestational age and perinatal death between groups.

Ethics and dissemination The methods used in this study are of low risk, but if successful, our findings will have a large impact on a large group of patients as infertility and asthma are the most common chronic diseases among the young population. The study has been approved by the Ethics Committee-Danish national research ethics committee $(\mathrm{H}-18016605)$ and the Danish Medicines Agency (EudraCT no: 2018-001137-41) and the Danish Data Protection Agency (journal number: VD2018486 and I-Suite number 6745). The test results will be published regardless of whether they are positive, negative or inconclusive. Publication in international peer-reviewed scientific journals is planned. Trial registration number NCT03727971.
Strengths and limitations of this study

- Large randomised controlled trial with 180 participants.

- Participants recruited from four different fertility clinics reducing selection bias.

- Double blinded.

- National study (only Denmark).

- Only allergic women with asthma.

\section{BACKGROUND}

Asthma is one of the most common chronic diseases among women of reproductive age characterised by both airway and systemic inflammation, reversible airway obstruction and airway hyper-responsiveness. The disease has a global prevalence of approximately 300 million patients. ${ }^{1}$ In the Western part of the world, asthma and infertility are among the most frequent chronic disorders in young adults (figure 1).

Several links have been established between asthma and the female reproduction as uncontrolled asthma both seems to be associated with a reduction in fertility and an increase in adverse perinatal outcomes. It has recently been shown that women with asthma have prolonged time to pregnancy $(\mathrm{TTP})^{2}$ and a tendency towards a higher number of miscarriages. ${ }^{3}$ Furthermore, a study, looking at asthma patients who recently had given birth to a full born child $(n=1106)$ and a matched control cohort $(n=4511)$, found that TTP was longer among asthmatics. ${ }^{4}$ These findings, in infertile asthma patients, suggest an inflammatory involvement of the reproductive organs preventing an optimal 




Figure 1 Prevalence of chronic disease among young adults. Figure adapted from 'Nielsen et al. ${ }^{31}$

implantation of the embryo. Maternal asthma has furthermore been associated with several pregnancy complications, such as gestational hypertension, pre-eclampsia, gestational diabetes and small for gestational age. ${ }^{5-8}$

\section{PREVIOUS RESEARCH}

Asthma affects pregnancy rate and thereby fertility by prolonging TTP. ${ }^{2} 3$ The negative effect of asthma on fertility increases with increasing age (above 35 years). ${ }^{9}$ Furthermore, the different respiratory microbiota ${ }^{10}$ or the difference in uterus and gut microbiota between normal and asthmatics might have significant importance for fertility. ${ }^{11}$

Unexplained infertile patients with asthma have lower values of vascular endothelial growth factor (VEGF) in the uterine cavity. ${ }^{12}$ This is of importance as high levels of VEGF are associated with successful pregnancy and twin pregnancies. ${ }^{1314}$ One hypothesis is that the concentration of VEGF in the lungs and the uterus is related to the microbiota, as previously reported based on samples from the intestinal tract of patients with chronic colitis. ${ }^{15}$ This could affect the receptiveness of the endometrium.

Non-atopic asthma is common among patients with adult-onset asthma and is often of neutrophilic phenotype. It is, however, unknown whether phenotypes of asthma, the eosinophilic (also referred to as T2 asthma) and the non-eosinophilic (often neutrophilic or non$\mathrm{T} 2$ ), are of importance for the fertility, as it has never been investigated. This could be the case as treatment response to inhaled corticosteroid (ICS) differs between phenotypes of asthma. T2 disease is characterised by increased eosinophilic airway inflammation. ${ }^{16}$ The treatment response to ICS is reduced in neutrophilic asthma (non-T2 disease), whereas the eosinophilic asthma patients, with T2 disease, are more sensitive to ICS. The different response to treatment, suggest a difference in the inflammatory mechanism, indicating that the phenotype might be of importance to fertility.

Treatment of allergic asthma with omalizumab works systemically by blocking the $\mathrm{IgE}$ receptor and reducing the systemic inflammation. ${ }^{17}$ Omalizumab is a humanised anti-IgE monoclonal antibody that selectively binds to free $\operatorname{IgE}$, which in turn decreases the level of $\operatorname{IgE}$ in the serum; it also reduces the number of high-affinity $\operatorname{IgE}$ receptors on mast cells, basophils and dendritic cells. This change in the mast cell degranulation decreases serum interleukin-31 levels, reversing basopenia and changing the basophil $\mathrm{IgE}$ receptor functions. ${ }^{18-20}$

The mechanism behind the association between asthma and infertility is still largely unknown. This study will hopefully give insight into this important question. The aim of our study was to determine if treatment with omalizumab prior to fertility treatment will increase pregnancy rate among women with asthma.

\section{METHODS}

\section{Objectives}

To explore differences in pregnancy rate between asthmatic women (with $\mathrm{IgE} \geq 75 \mathrm{kU} / \mathrm{L}$ ) receiving both standard asthma treatment and omalizumab treatment versus asthmatic women receiving standard asthma treatment and placebo. Pregnancy rate is defined as ongoing pregnancy confirmed with ultrasound in gestational week 7 , after one of three consecutive embryo transfers, both fresh and frozen embryos.

\section{Primary outcome}

To compare pregnancy rates in asthma patients undergoing in vitro fertilisation (IVF)/intracytoplasmatic sperm injection (ICSI) treatment when treated with either omalizumab or placebo in up to three consecutive fertility treatments with embryo transfer.

\section{Secondary outcomes}

A. Change in sputum and blood eosinophil cell count (before and after treatment).

B. Change in VEGF, $\mathrm{C}$ reactive protein (CRP), interleukin-6 (IL-6), interleukin-8 (IL-8), IgE, tumour necrosis factor alpha (TNFa), eosinophil cationic protein (ECP) in blood, sputum and uterus secretion (before and after treatment).

C. Changes in the microbiota in sputum, the vagina, the uterus and the rectum (before and after treatment).

D. Rate of pregnancy loss, that is, difference between positive s-hCG (>3 IU/L) day 14 and ongoing pregnancy gestational week 7 , gestational week 12 and birth.

E. Number of malformations, preterm, pre-eclampsia, perinatal death, Small for Gestational Age (SGA), birth weight in both groups.

\section{STUDY DESIGN, RANDOMISATION AND INTERVENTION}

The investigational medicinal product (IMP)—omalizumab have been manufactured and marketed with the indication severe allergic asthma since 2005 and later also for chronic spontaneous urticaria. In this study, we will investigate the effect of omalizumab on infertility among women with asthma, which at present is not an indication 


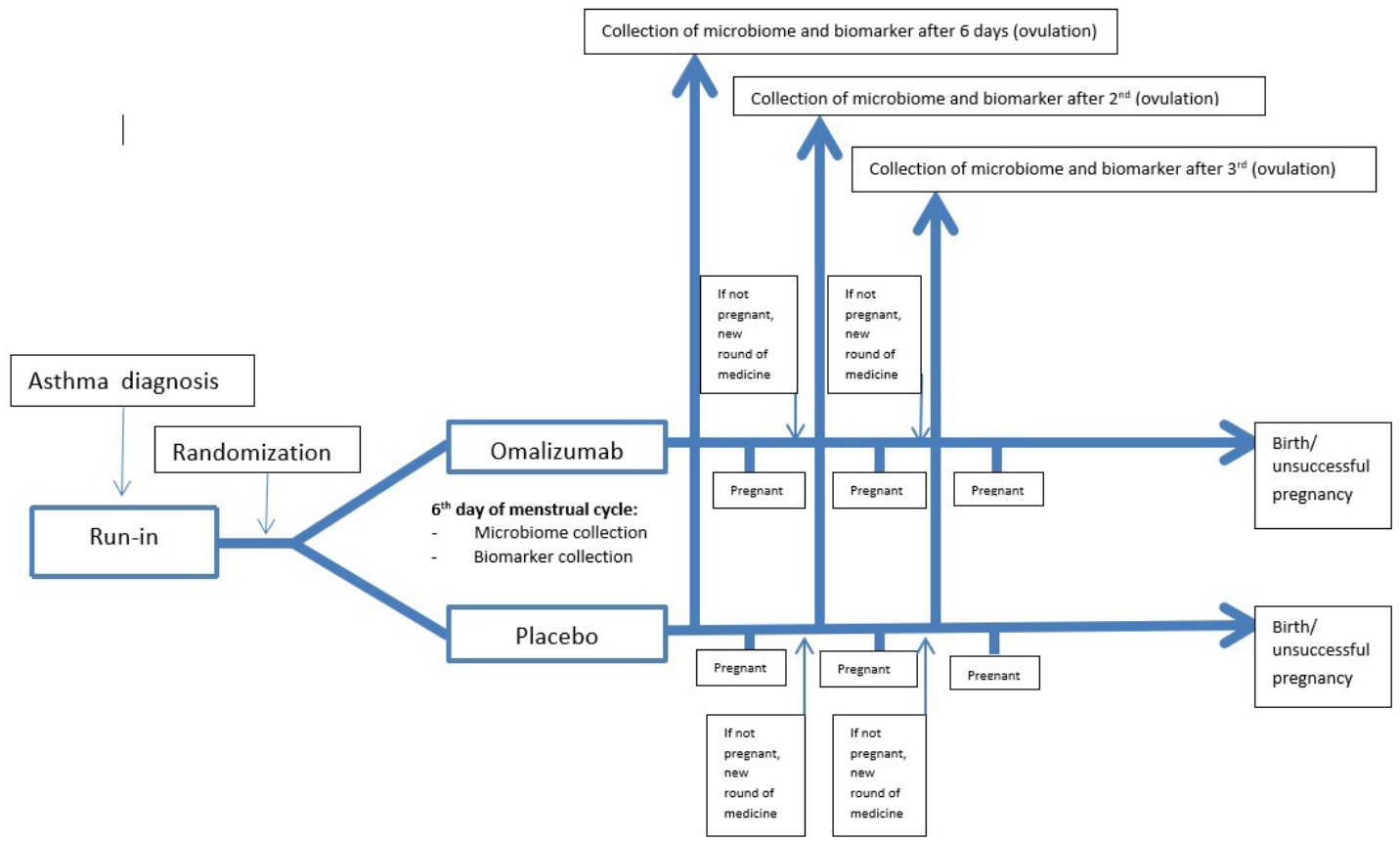

Figure 2 Flowchart of patients through the study.

for the IMP. Therefore, the present study will be run as a phase IIa study, that is, proof-of-concept study (figure 2).

This protocol is a randomised, double-blind, parallel group, study to evaluate the difference between omalizumab $(\mathrm{O})$ and placebo $(\mathrm{P})$ on pregnancy rate in patients with allergic asthma. Treatment schedule (table 1):

- At enrolment, the subjects will have 1-2 weeks of run-in (diagnosis of asthma) with collection of questionnaire data and baseline values. No collection of biomaterial will be done at the time of enrolment, as this will be on different time points of the female cycles.

The subjects are randomised to either the omalizumab treatment group or the placebo group by a computerbased programme blinded to all investigators. Patients will be randomised in blocks of 10 , and 16 blocks in total. It will be an automated random assignment of subject numbers to randomisation numbers. These randomisation numbers are linked to different treatment arms, which in turn are linked to medication numbers.

- On day $6( \pm 1$ day) of the menstrual cycle, biomaterials are collected (blood samples, sputum, secretion of the vagina, secretion of the rectum, microbiota).

- The omalizumab or placebo treatment is administered as one injection of weight and se-IgE balanced omalizumab or one injection of placebo on the sixth day $( \pm 1$ day) of the menstrual cycle after collection of the biomaterial.

- After omalizumab treatment and in-between egg aspiration and embryo transfer, biomaterial (blood samples, sputum, secretion of the vagina, secretion of the rectum, secretion of the uterus, microbiota) will be collected once more. If no pregnancy has occurred after the first embryo transfer, the omalizumab or placebo treatment will be repeated for additional two cycles with IVF/ICSI.
- Pregnant subjects are followed up until delivery or pregnancy loss.

- The subjects will be followed up 1 year after the last embryo transfer.

\section{Selection of participants}

At the first consultation at the fertility clinic, the potential participants will be given a questionnaire with 20 standardised European Academy of Allergy and Clinical Immunology (EAACI) clinical questions regarding asthma and allergy (validated as an asthma and allergy screening tool). If the women respond positively to one or more questions of the questionnaire, they will be contacted and screened for the presence of asthma and possible inclusion in the project.

This study will enrol females, 18-40 years of age with stable asthma with an ACQ $\leq 1.5$. The subjects must have a total IgE $\geq 75 \mathrm{kU} / \mathrm{L}$ or/and a positive skin prick test or specific IgE of standard aeroallergens. The treatment at the time of enrolment should be The Global Initiative for asthma (GINA) guidelines step 1 to step 4 , which is short-acting beta2-agonist (SABA) with or without controller treatment with ICS, and lastly, additional second controller with montelukast or long-acting beta2-agonist, if needed (GINA 1-4). ${ }^{1}$ In case of uncontrolled asthma (ie, ACQ $>1.5$ ), inclusion will be postponed for 4weeks, in which period asthma treatment will be optimised and the subjects can be re-screened for enrolment when $\mathrm{ACQ} \leq 1.5$ over the last 2 weeks.

Women eligible for the study must be infertile due to tubal factor infertility or unexplained infertility and/or the partner has male factor infertility. Women with other causes of infertility are not eligible for inclusion in the study.

\section{Inclusion criteria}

- Informed consent (online supplemental file 1). 


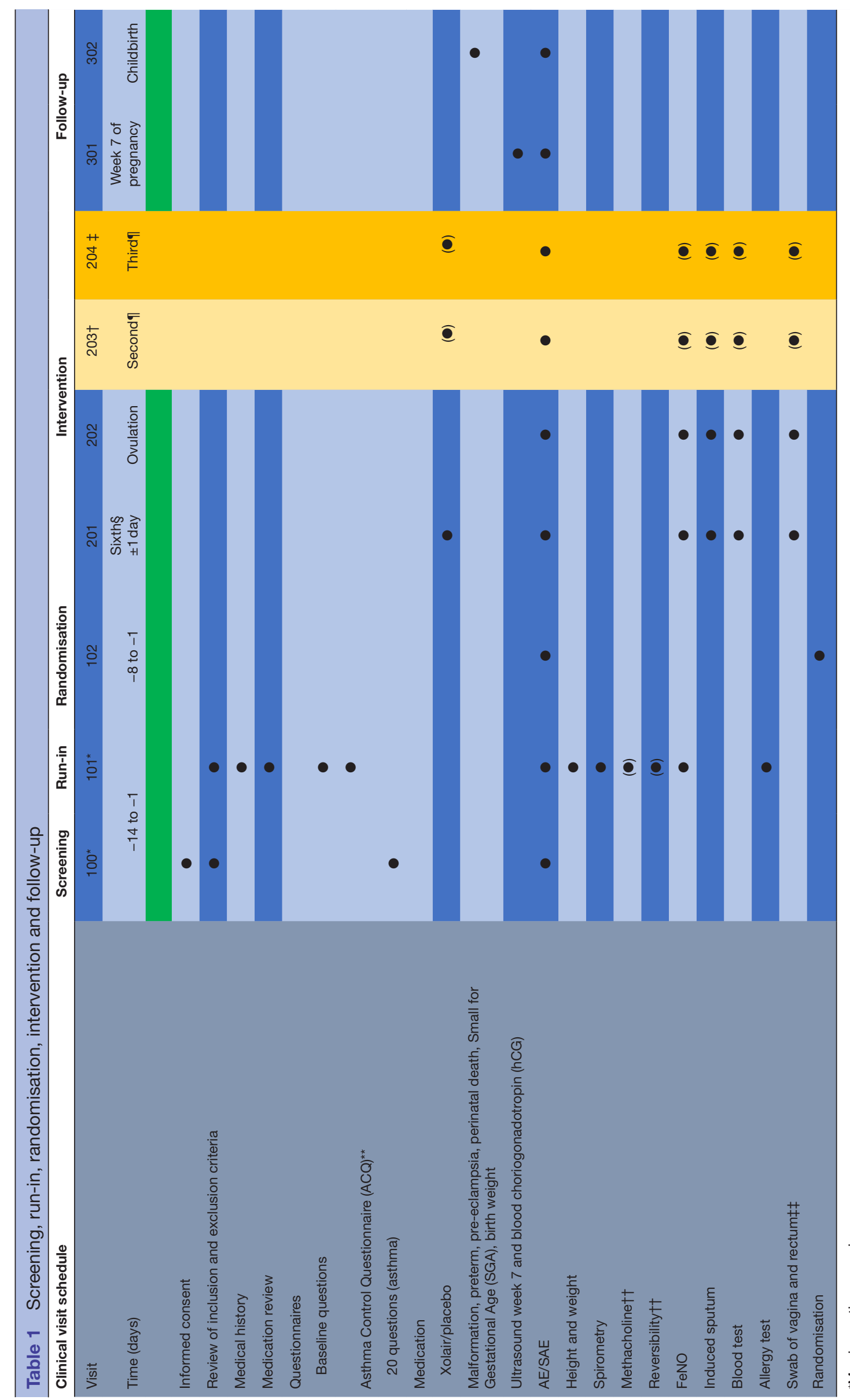


- Diagnosis of asthma (objectively verified at enrolment).

- Total $\operatorname{IgE} \geq 75 \mathrm{kU} / \mathrm{L}$ or/and a positive skin prick test or specific IgE to standard aeroallergens.

- Infertility due to male factor infertility, tubal factor infertility or unexplained infertility.

- Referred to IVF treatment with or without ICSI.

- Willingness to receive treatment with biologic drug.

- Controlled disease with an ACQ $\leq 1.5$.

\section{Exclusion criteria}

- Other respiratory diseases than asthma.

- Other inflammatory disease or a disease that affects fertility.

- Allergy to the investigational drugs.

- Respiratory infections requiring antibiotics or antiviral treatment within 30 days.

- Current smokers and tobacco consumption more than 10 pack-years.

- Testicular sperm aspiration/testicular sperm extraction, polycystic ovary syndrome, endometriosis.

- Infertility due to other reasons than male factor, tubal factor or unexplained infertility.

\section{Methods for ensuring blinding}

All packaging and labelling of the biological drugs and placebo will be done in a way that ensures blinding for the investigator site staff. Herlev Apotek, the hospital pharmacy at the Capital Region, will manage randomisation and blinding. Injection of the study drug/placebo will be administered by a unblinded study nurse who have no affiliations to the study.

\section{MEASUREMENTS}

\section{Basic values}

At the time of enrolment, weight, height and pregnancy status (s-hCG) will be measured in all patients.

\section{Lung function measurement}

Spirometry and lung volume (total lung capacity (TLC), residual volume $(\mathrm{RV})$ ) with Jaeger Spirometer will be used for lung function measurement.

\section{Bronchial challenge}

Bronchial challenge will be done using inhaled methacholine. ${ }^{21}$ In case of negative methacholine test and clinical signs of asthma, mannitol test will be performed. Response-dose ratio in both methacholine and mannitol will be calculated. In case of forced expiratory volume in one second $<70 \%$ prebronchial challenge, reversibility test will be performed as described below.

\section{Reversibility test for short-acting beta2}

A spirometry in accordance with international standards as described above is initially performed. Following the pulmonary function test, a standard dose of SABA (400 $\mu \mathrm{g}$ salbutamol or $1 \mathrm{mg}$ terbutaline) is administered and the spirometry is repeated after approximately $15 \mathrm{~min}$.

\section{Fraction of exhaled nitric oxide (FeNO)}

The subject inhales air without NO, followed by a $10 \mathrm{~s}$ exhalation maintaining a constant flow rate of $50 \mathrm{~mL} / \mathrm{s}$. Test will be performed three times and the mean concentration registered. ${ }^{22}$

\section{Microbiota and inflammation \\ Induced sputum}

The sputum is performed with hypertonic saline in nebuliser. The induction was performed at $5 \mathrm{~min}$ intervals for $\leq 15 \mathrm{~min}$. Women were instructed to cough and spit after 5, 10 and 15 min of induction or whenever they got the urge to do so. It was collected with the aim of differential count, markers of inflammation and microbiota.

\section{Vaginal smear}

Swabs will be obtained at day 6 and in-between egg aspiration and embryo transfer. A vaginal smear for microbiota has been developed for self-administrative purpose. Patients will receive a kit.

\section{Rectal smear}

Swabs will be obtained at day 6 and in-between egg aspiration and embryo transfer. A rectal smear for microbiota has been developed for self-administrative purpose. Patients will receive a kit.

\section{Mouth smear}

Swabs will be obtained at day 6 and in-between egg aspiration and embryo transfer. A mouth smear for microbiota has been developed for self-administrative purpose. Patients will receive a kit.

\section{Blood samples}

A total of $200 \mathrm{~mL}$ of blood, $40 \mathrm{~mL}$ per visit, will be taken, it will be used for analyses of VEGF, CRP, IL-6, IL-8, IgE, TNFa and ECP.

\section{Questionnaires}

The participants will be answering several questions, 'astma \& allergi spørgeskema', mini-asthma quality of life questionnaire (AQLQ), Nijmegen, ACQ 5 and hospital anxiety and depression scale (HADS).

\section{Withdrawal of study}

A subject can be excluded from the study if the subject is not able to adhere to the study protocol. This should be confirmed by the coordinating investigator. All subjects are allowed to withdraw during the study, if subjects wish to do so.

\section{Size of the study population and statistical considerations}

In a group of asthma patients to test, an omalizumab induced reduction in number of cycles of fertility treatment to evaluate pregnancy rate per embryo transfer. Treatment with omalizumab indicated $41 \%$ pregnancy rate (omalizumab) to $19 \%$ pregnancy rate (placebo) after three cycles of fertility treatment (s-hCG test after 2 weeks and ongoing pregnancy ultrasound after 7 weeks) and followed for 1 year. Secondary outcomes are 
ultrasound-confirmed clinical pregnancies ongoing after 3 months, baby take home rate and the rate of complication. With a $\mathrm{p}$ value of 0.05 and a power of 0.8 , a group of 72 patients with allergic asthma should be included in each arm; regulating for drop-out (20\%), 90 allergic asthma patients in each arm. Recruitment will be stopped when 72 patients in each arm have become pregnant or completed three cycles of IVF.

\section{Stratification}

Stratification will be based on blood eosinophilic cell count with a cut-off $0.3 \mathrm{mia} / \mathrm{L}$, ensuring equal number of eosinophilic and non-eosinophilic asthma patients in each group. We will also stratify for age, with a cut-off $>30$ years, ensuring equal number of patients with high and low age in each group. We will also stratify for male factor infertility and tubal factor to ensure equal number in each group

We based the sample size calculation on the log rank test using Freedman's equation, ${ }^{23}$ a power at $80 \%$, a significance level at $5 \%$ and assumed equal number of participants in the intervention and control group, who all will be followed for 1 year. Based on a previous observational study, we estimated that $19 \%$ in the placebo group will be pregnant within a year. ${ }^{2}$ If we want to be able to detect a HR at 2,5, which corresponds to $41 \%$ getting pregnant in the intervention group, we need 72 patients in each group. Given an assumption of a $20 \%$ drop out rate, a total of 180 patients will have to be recruited.

The findings of the study will be analysed and presented using descriptive statistics reporting on various demographic data. To calculate findings related to the primary endpoint 'pregnancy rate', a side-by-side (such as unmatched t-test) analysis will be applied. To calculate the multiple secondary endpoints in the study, regression analysis will be applied. In addition, an analysis for non-detected confounding variables will be conducted (such as a two-stage sampling).

\section{Patient and public involvement}

Patients or the public will be involved in the reporting and dissemination of our research.

In addition to giving written feedback to all study participants, we will send all our reports and publications to the relevant patient organisation. Patients and/ or public were not involved in the design of the study.

\section{Trial Steering Committee and Data Safety Monitoring Board}

A Data Safety Monitoring Board (unblinded) is in place to closely and independently monitor the safety of the PROART study.

\section{DISCUSSION}

To date, several studies have shown increased TTP and a higher need for fertility treatment among women with asthma. The mechanism behind is still unclear and partly unexplored. There are speculations regarding the inflammation that is known to be prominent in the lungs of asthma patients, not only being limited to the lungs but also systemic and possibly prominent in the uterine lining resulting in an imbalance that might impair implantation. ${ }^{2425}$ Acknowledging that achieving pregnancy can be challenging for women with asthma, this study is setting out to investigate whether omalizumab can lower the systemic and local inflammation and thereby increase the pregnancy success rate in a randomised controlled trial.

This study may help us understand the underlying mechanisms of asthma as a systemic disease, as well as exploring treatment options for women with asthma with difficulties becoming pregnant. The methods used in this study are of low risk, but if successful, our findings will have a large impact on a large group of patients as infertility and asthma are the most common chronic diseases among the young population. ${ }^{26}$ There are a few studies looking into the safety of omalizumab during pregnancy. However, both studies conclude that it is safe to use omalizumab during pregnancy. ${ }^{27}{ }^{28}$ Also, some case studies have reported no adverse events related to treatment with omalizumab during pregnancy. ${ }^{29} 30$

The hypothesis is that increased asthma control with biological treatment reduces local and systemic inflammation, which may improve the pregnancy success rate, adverse perinatal outcomes and other known pregnancy complications. We propose that targeting both the local and systemic inflammation with immune-modifying therapy, such as biologic drugs, can improve fertility.

An increase in pregnancy rate as well as reduction in TPP is important for the individual women and her partner, as well as the society. In order to provide recommendations for women with asthma, who have difficulties becoming pregnant, there is a need for a clinical trial testing a systemic therapy.

\section{Trial status}

Patient recruitment commenced in January 2019 and is ongoing.

\section{ETHICS AND DISSEMINATION}

The study will be carried out to include the protection of human subjects according to the 2008 Declaration of Helsinki and in accordance with Good Clinical Practice Guidelines. Patients will be informed about the study at first visit and included if accepting to participate. A completed patient informed consent form is required from all patients participating in the study and must be signed by the patient and the informing physician. The study has been approved by the Ethics Committee-Danish national research ethics committee (H-18016605) and the Danish Medicines Agency (EudraCT no: 2018-001137-41) and the Danish Data Protection Agency (journal number: VD-2018486 and I-Suite number 6745).

The test results will be published regardless of whether they are positive, negative or inconclusive. Publication in international peer-reviewed scientific journals is planned 
and accompanied by parallel publications in Danish Medical Journal. We aim to publish in high impact scientific journals.

Results that cannot be published in peer-reviewed journals will be published in congresses in the form of posters and oral presentations.

\section{Author affiliations}

${ }^{1}$ Department of Respiratory Diseases, Hvidovre Hospital, University of Copenhagen, Copenhagen, Denmark

${ }^{2}$ Department of Obstetrics and Gynecology, Roskilde Hospital, Copenhagen, Denmark

${ }^{3}$ Department of Obstetrics and Gynaecology, The Fertility Clinic, Copenhagen University Hospital, Hvidovre Hospital, Copenhagen, Denmark

${ }^{4}$ Fertility Clinic, Copenhagen University Hospital, Copenhagen, Denmark

${ }^{5}$ Laboratory of Genomics and Molecular Biomedicine, Department of Biology,

University of Copenhagen, Copenhagen, Denmark

${ }^{6}$ Fertility Clinic, Herlev Hospital, Herlev, Denmark

${ }^{7}$ Centre for Physical Activity Research, Rigshospitalet, Kobenhavn, Denmark

Acknowledgements Many thanks to all the involved fertility clinics at the Capital Region of Denmark for allowing us to recruit patients.

Contributors CT prepared the study design and conducted the first draft protocol in collaboration with VB. When read, edited and approved by the co-authors (EJG, HSN, KK, NLCF and CSU), CT applied for approvals at the Ethics Committee, Danish Medicines Agency and the Danish Data Protection Agency. CT and VB applied for grants at Novartis. CT registered the trial at clinicaltrials.gov. All authors (EJG, CSU, HSN, BSO-M, KK, NLCF, BN, HU, VB and CT) have read, edited and approved the final manuscript.

Funding The study is financed by a grant from Novartis, and the study drug omalizumab is provided by Novartis - Award/grant number: CIGE025ADK01T PROART.

Competing interests None declared.

Patient consent for publication Not required.

Provenance and peer review Not commissioned; externally peer reviewed.

Supplemental material This content has been supplied by the author(s). It has not been vetted by BMJ Publishing Group Limited (BMJ) and may not have been peer-reviewed. Any opinions or recommendations discussed are solely those of the author(s) and are not endorsed by BMJ. BMJ disclaims all liability and responsibility arising from any reliance placed on the content. Where the content includes any translated material, BMJ does not warrant the accuracy and reliability of the translations (including but not limited to local regulations, clinical guidelines, terminology, drug names and drug dosages), and is not responsible for any error and/or omissions arising from translation and adaptation or otherwise.

Open access This is an open access article distributed in accordance with the Creative Commons Attribution Non Commercial (CC BY-NC 4.0) license, which permits others to distribute, remix, adapt, build upon this work non-commercially, and license their derivative works on different terms, provided the original work is properly cited, appropriate credit is given, any changes made indicated, and the use is non-commercial. See: http://creativecommons.org/licenses/by-nc/4.0/.

ORCID iD

Casper Tidemandsen http://orcid.org/0000-0001-6032-6414

\section{REFERENCES}

1 pp. Becker AB, Abrams EM. Asthma guidelines: the global initiative for asthma in relation to national guidelines. Curr Opin Allergy Clin Immunol 2017;17:99-103.

2 Gade EJ, Thomsen SF, Lindenberg S, et al. Fertility outcomes in asthma: a clinical study of 245 women with unexplained infertility. Eur Respir J 2016;47:1144-51.

3 Gade EJ, Thomsen SF, Lindenberg S, et al. Asthma affects time to pregnancy and fertility: a register-based twin study. Eur Respir J 2014;43:1077-85.

4 Grzeskowiak LE, Smithers LG, Grieger JA, et al. Asthma treatment impacts time to pregnancy: evidence from the International scope study. Eur Respir J 2018;51:1702035.
5 Johnston S, Said J. Perinatal complications associated with maternal asthma during pregnancy. Obstet Med 2012;5:14-18.

6 Martel M-J, Rey Évelyne, Beauchesne M-F, et al. Use of inhaled corticosteroids during pregnancy and risk of pregnancy induced hypertension: nested case-control study. BMJ 2005;330:230-3.

7 Baghlaf H, Spence AR, Czuzoj-Shulman N, et al. Pregnancy outcomes among women with asthma. J Matern Fetal Neonatal Med 2019;32:1325-31.

8 Ali Z, Nilas L, Ulrik CS. Low risk of adverse obstetrical and perinatal outcome in pregnancies complicated by asthma: a case control study. Respir Med 2016;120:124-30.

9 Vejen Hansen A, Ali Z, Malchau SS, et al. Fertility treatment among women with asthma: a case-control study of 3689 women with live births. Eur Respir J 2019;53:1-3.

10 Sverrild A, Kiilerich P, Brejnrod A, et al. Eosinophilic airway inflammation in asthmatic patients is associated with an altered airway microbiome. J Allergy Clin Immunol 2017;140:407-17.

11 Schoenmakers S, Steegers-Theunissen R, Faas M. The matter of the reproductive microbiome. Obstet Med 2019;12:107-15.

12 Gade EJ, Thomsen SF, Lindenberg S, et al. Lower values of VEGF in endometrial secretion are a possible cause of subfertility in nonatopic asthmatic patients. J Asthma 2015;52:336-42.

13 Evans PW, Wheeler T, Anthony FW, et al. A longitudinal study of maternal serum vascular endothelial growth factor in early pregnancy. Hum Reprod 1998;13:1057-62.

14 Seo WS, Jee BC, Moon SY. Expression of endometrial protein markers in infertile women and the association with subsequent in vitro fertilization outcome. Fertil Steril 2011;95:2707-10.

15 Wang X, Zhao J, Qin L. Vegf-C mediated enhancement of lymphatic drainage reduces intestinal inflammation by regulating IL-9/IL-17 balance and improving gut microbiota in experimental chronic colitis. Am J Transl Res 2017;9:4772-84.

16 Kuruvilla ME, Lee FEH, Lee GB, et al. Endotypes, and mechanisms of disease. Clinical Reviews in Allergy and Immunology 2019;56:219-33.

17 Bousquet J, Siergiejko Z, Świebocka E, et al. Persistency of response to omalizumab therapy in severe allergic (IgE-mediated) asthma. Allergy 2011;66:671-8.

18 Beck LA, Marcotte GV, MacGlashan D, et al. Omalizumab-induced reductions in mast cell FCE psilon RI expression and function. $J$ Allergy Clin Immunol 2004;114:527-30.

19 Saini SS, MacGlashan DW, Sterbinsky SA, et al. Down-Regulation of human basophil IgE and Fc epsilon RI alpha surface densities and mediator release by anti-lgE-infusions is reversible in vitro and in vivo. J Immunol 1999;162:5624-30.

20 Macglashan DW, Saini SS. Omalizumab increases the intrinsic sensitivity of human basophils to IgE-mediated stimulation. J Allergy Clin Immunol 2013;132:906-11.

21 Sue-Chu M, Brannan JD, Anderson SD, et al. Airway hyperresponsiveness to methacholine, adenosine 5-monophosphate, mannitol, eucapnic voluntary hyperpnoea and field exercise challenge in elite cross-country skiers. Br J Sports Med 2010;44:827-32.

22 Kharitonov SA, Walker L, Barnes PJ. Repeatability of standardised nasal nitric oxide measurements in healthy and asthmatic adults and children. Respir Med 2005;99:1105-14.

23 Freedman LS. Tables of the number of patients required in clinical trials using the logrank test. Stat Med 1982;1:121-9.

24 Feng $\mathrm{CH}$, Miller MD, Simon RA. The United allergic airway: connections between allergic rhinitis, asthma, and chronic sinusitis. Am J Rhinol Allergy 2012;26:187-90.

25 Holt PG, Sly PD. Interaction between adaptive and innate immune pathways in the pathogenesis of atopic asthma: operation of a lung/ bone marrow axis. Chest 2011;139:1165-71.

26 Andersen A-M, Nielsen HS. Fertilitet og sundhed," in Fosterlivet: Farefuldt og fantastiske fremtidsudsigter 2012:59-82.

27 Namazy JA, Blais L, Andrews EB, et al. Pregnancy outcomes in the omalizumab pregnancy registry and a disease-matched comparator cohort. J Allergy Clin Immunol 2020;145:528-36.

28 Kupryś-Lipińska I, Tworek D, Kuna P. Omalizumab in pregnant women treated due to severe asthma: two of good outcomes of pregnancies. Pdia 2014;2:104-7.

29 Ensina LF, Cusato-Ensina AP, Camelo-Nunes IC, et al. Omalizumab as third-line therapy for urticaria during pregnancy. J Investig Allergol Clin Immunol 2017;27:326-7.

30 Malara G, Sciarrone C. Chronic spontaneous urticaria treated with omalizumab: report of two cases. J Dermatolog Treat 2018;29:3-5.

31 Nielsen HS, Schmidt L, Nyboe Andersen A, et al. Forebyggelse $A F$ nedsat frugtbarhed. København: Vidensråd for forebyggelse, 2016: s.23. 